\title{
On a convective condition in the diffusion of a solvent into a polymer
}

\author{
MARCOS GAUDIANO $^{1}$, TOMÁS GODOY ${ }^{2}$ and CRISTINA TURNER ${ }^{1,2}$ \\ ${ }^{1}$ FAMAF-UNC-CIEM-CONICET \\ ${ }^{2}$ FAMAF-UNC. Córdoba, Argentina \\ E-mails: gaudiano@mate.uncor.edu / godoy@mate.uncor.edu / turner@mate.uncor.edu
}

\begin{abstract}
We studied a one-dimensional free boundary problem arising in the polymer industry, which solution has an interesting asymptotic behavior when a convective boundary condition is imposed. We show the asymptotic behavior of the free boundary and of the concentration of the solvent in the domain, for large $t$. Exact estimates and numerical results are obtained.
\end{abstract}

Mathematical subject classification: 35K05, 35K60.

Key words: free boundary problems, diffusion, convective case, asymptotic behavior.

\section{Introduction}

In this paper we consider a free boundary problem arising from a model for sorption of solvents into glassy polymers.

This model was proposed in [1] by Astarita and Sarti. They assumed that the sorption process can be described using a free boundary model to simulate a sharp morphological discontinuity observed in the material between a penetrated zone, with a relatively high solvent content, and an glassy region where the solvent concentration is negligibly small (and actually taken to be zero in the model).

The solvent is supposed to diffuse in the penetrated zone according to Fick's law. Moreover the penetrating zone moves into the glassy zone driven by chemical and mechanical effects that are taken into account by an empirical law relating the speed of penetration to the concentration of solvent near the front. This law 
must account for two main facts observed in the penetration experiences: (i) there exists a threshold value for the solvent concentration under which no penetration occurs; (ii) above such value the speed of the front increases with the concentration near the front itself. A typical form is $v=\alpha|u-q|^{m}$ where $v$ is the front speed, $u$ is the value of the concentration at the front, $q>0$ is the threshold value and $\alpha$ and $m$ are positive constants ([1]).

An additional condition on the free boundary is obtained imposing mass conservation, i.e., equating the mass density current to the product of solvent concentration and the velocity of the free boundary.

This model has been the object of a number of papers. In [2], it has been studied with the condition of constant concentration at the boundary. In [3], it has been investigated assuming that the polymer is in perfect contact with a well-stirred bath. In [4], its authors were interested in the case of a slab of nonhomogeneous polymer. In [5], it has been studied assuming a flux condition at the fixed boundary. Here we are interested in a convective case, where it is supposed that there is a flux of solvent through the left side of a slab proportional to the difference between the solvent concentration at $x=0$ and a given function of the time which represents an external solvent concentration $(h>0$ is the proportionality constant). Denoting by $c(x, t)$ the normalized concentration and by $x=s(t)$ the location of the front in the slab the mathematical problem can be stated as follows:

Problem PS. Find a triple $(T, s, c)$ such that: $T>0, s \in C^{1}[0, T], c \in$ $C^{2,1}\left(D_{T}\right) \cap C\left(\bar{D}_{T}\right)$, where $D_{T}=\{(x, t): 0<t<T, 0<x<s(t)\}$, and satisfying

$$
\begin{aligned}
& c_{x x}-c_{t}=0 \quad \text { in } \quad D_{T}, \\
& c_{x}(0, t)=h[c(0, t)-g(t)], \quad g(0)=1, \quad 0 \leq t \leq T \\
& \dot{s}(t)=f(c(s(t), t)), \quad 0 \leq t \leq T \\
& c_{x}(s(t), t)=-\dot{s}(t)[c(s(t), t)+q], \quad 0 \leq t \leq T \\
& s(0)=0 .
\end{aligned}
$$

The function $g(t)$ is positive and the quantity $q+g(t)$ represents the external concentration. In order to assure a stable process we suppose that $g \in C^{1}[0, T]$, 
$\forall T>0, g^{\prime}(t) \leq 0$ and $G \equiv \int_{0}^{\infty} g(t) d t<\infty$. Throughout the paper the function $f$ will be supposed to satisfy $f \in C^{1}(0,1], f^{\prime}(c)>0$ for $c \in(0,1]$ and $f(0)=0$ (empirically, the function $f$ is observed to be a power law). We note that there exists $\Phi=f^{-1}$ which has the same properties as $f$.

\section{Existence and some global estimates}

Equating (1.2) to (1.4) for $t=0$, we have that $c^{*} \doteq c(0,0)$ is the unique solution of $f\left(c^{*}\right)\left(c^{*}+q\right)=-h\left(c^{*}-1\right)$. The solution satisfies $0<c^{*}<1$.

The existence for PS is accomplished as follows: Let $r \in C^{1}[0, T] \cap C^{2}(0, T)$ be such that

$$
\begin{aligned}
& r(0)=0, \\
& \dot{r}(0)=f\left(c^{*}\right), \\
& 0 \leq \dot{r}(t) \leq f\left(c^{*}\right) \quad \text { in } \quad[0, T], \\
& |\ddot{r}(t)| \leq K \quad \text { in } \quad(0, T),
\end{aligned}
$$

and consider the problem (PA) of finding $c \in C^{2,1}(D) \cap C(\bar{D}), c_{x}$ continuous up to $x=r(t), t \in(0, T)$, such that

$$
\begin{aligned}
& c_{x x}-c_{t}=0 \quad \text { in } \quad D=\{(x, t): 0<t<T, 0<x<r(t)\}, \\
& c_{x}(0, t)=h[c(0, t)-g(t)], \quad g(0)=1, \quad 0 \leq t \leq T, \\
& c_{x}(r(t), t)=-\dot{r}(t)[\Phi(\dot{r}(t))+q], \quad 0 \leq t \leq T .
\end{aligned}
$$

Thus, we note that PA differs from PS by the fact that the curve $r(t)$ is given. In [7], it is shown that the transformation

$$
r(t) \rightarrow \int_{0}^{t} f(c(r(t), t)), \quad 0<t<T
$$

has a unique fixed point for $T>0$ small enough, which is actually the desired curve $s(t)$ of PS. The global existence and uniqueness for PS is also established in [7]. Now we prove:

Proposition 2.1. Assume $s, c$ solve problem $\mathbf{P S}$ for a given $T<+\infty$. Then

$$
\begin{array}{lll}
0<c(x, t)<1 & 0 \leq x \leq s(t), & t \geq 0, \\
h\left(c^{*}-1\right)<c_{x}(x, t)<h(1-g(t)) & 0 \leq x \leq s(t), & t>0,
\end{array}
$$


Proof. Using the Hopf's lemma we can assume that $c$ attains its maximum value on $x=0$ since $c_{x}=-f(c)(\Phi(\dot{s})+q) \leq 0$ on $x=s(t)$. Let be $c\left(0, t_{0}\right)=\max _{D_{T}} c$. If $t_{0}=0$, then $\max _{D_{T}} c=c^{*}<1$. Otherwise we have $0>c_{x}\left(0, t_{0}\right)=h\left(c\left(0, t_{0}\right)-g\left(t_{0}\right)\right)$, which implies $\max _{D_{T}} c<g\left(t_{0}\right) \leq 1$. Let be $c\left(x_{1}, t_{1}\right)=\min _{D_{T}} c$. If $x_{1}=0$ then there occurs either $\min _{D_{T}} c=c^{*}>0$ or $\min _{D_{T}} c=g\left(t_{1}\right)+1 /(h) c_{x}\left(0, t_{1}\right) \geq g\left(t_{1}\right)>0$. Moreover, if $\min _{D_{T}} c=$ $c\left(s\left(t_{1}\right), t_{1}\right)=0$ (with $t_{1}>0$ ) then $c_{x}\left(s\left(t_{1}\right), t_{1}\right)=0$, contradicting the boundary point principle. Thus, (2.14) holds and $c_{x}(0, t)<h(1-g(t))$ for all $t$. Finally, let us suppose that $\min _{D_{T}} c_{x}=c_{x}\left(s\left(t_{2}\right), t_{2}\right)$ with $t_{2} \geq 0$. If $t_{2}>0$ then

$$
\begin{aligned}
0 \geq & \left.\frac{d}{d t} c_{x}(s(t), t)\right|_{t=t_{2}}, \quad \text { i.e. } \\
0 \leq- & {\left[f^{\prime}\left(c\left(s\left(t_{2}\right), t_{2}\right)\right)\left(c\left(s\left(t_{2}\right), t_{2}\right)+q\right)+f\left(c\left(s\left(t_{2}, t_{2}\right)\right)\right)\right] } \\
& {\left[c_{x}\left(s\left(t_{2}\right), t_{2}\right) \dot{s}\left(t_{2}\right)+c_{x x}\left(s\left(t_{2}\right), t_{2}\right)\right]>0, }
\end{aligned}
$$

which is a contradiction. Then $\min _{D_{T}} c_{x}=c_{x}(0,0)=h\left(c^{*}-1\right)$.

Proposition 2.2. Under the assumptions above, the following estimate holds

$$
\left|c_{t}(x, t)\right| \leq B_{T}, \quad \forall(x, t) \in D_{T},
$$

with

$$
B_{T}=\max \left\{\max _{[0, T]}\left|g^{\prime}\right|, f(1)^{2}(1+q),\left|c_{t}(0,0)\right|\right\}
$$

Proof. Note from (1.2) that

$$
c_{t}(0, t)=g^{\prime}(t)+\frac{1}{h} c_{t x}(0, t), \quad 0<t \leq T .
$$

Moreover, from (1.3) and (1.4) we have $c_{x}=-f(c)(c+q)$ at $x=s(t)$ and deriving with respect to $t$ we get

$$
\begin{aligned}
& c_{t} f(c)+c_{t x}=-\left[f^{\prime}(c)(c+q)+f(c)\right]\left(c_{x} f(c)+c_{t}\right) \text { at } x=s(t) \\
& c_{t}(s(t), t)=\left(\frac{\left[f^{\prime}(c)(c+q)+f(c)\right] f^{2}(c)(c+q)-c_{t x}}{2 f(c)+f^{\prime}(c)(c+q)}\right)_{x=s(t)}
\end{aligned}
$$

The proposition follows by using the Hopf's lemma. 


\section{The numerical method}

In this section will be shown a numerical scheme based on the method introduced in [6] for one-dimensional parabolic free boundary problems with arbitrary implicit or explicit free boundary conditions.

In this method the continuous problem is time discretized and solved at successive time levels as a sequence of free boundary problems for ordinary differential equations. Specifically, at time level $t=t_{n}$ with $t_{n}-t_{n-1}=\Delta t$ the solution $\left\{C_{n}(x), S_{n}\right\}$ is computed as the exact solution of the discretized equations

$$
\begin{aligned}
& C_{n}^{\prime \prime}-\frac{C_{n}-C_{n-1}}{\Delta t}=0 \quad 0<x<S_{n}, \\
& C_{n}^{\prime}(0)=h\left(C_{n}(0)-g\left(t_{n}\right)\right), \\
& \frac{S_{n}-S_{n-1}}{\Delta t}=f\left(C_{n}\left(S_{n}\right)\right), \quad S_{0}=0, \\
& C_{n}^{\prime}\left(S_{n}\right)=-\frac{S_{n}-S_{n-1}}{\Delta t}\left(q+C_{n}\left(S_{n}\right)\right) .
\end{aligned}
$$

In (3.21) the function $C_{n-1}(x)$ is supposed to be defined over $[0,+\infty)$, and $S_{n-1}$ supposed to be known as well. We write (3.21) as a first order system over $\left(0, S_{n}\right)$

$$
\begin{aligned}
C_{n}^{\prime} & =V_{n}, \\
V_{n}^{\prime} & =\frac{1}{\Delta t}\left(C_{n}-C_{n-1}\right)
\end{aligned}
$$

and exploit the observation that $C_{n}$ and $V_{n}$ are related through the Riccati transformation

$$
C_{n}(x)=R(x) V_{n}(x)+W_{n}(x),
$$

where

$$
\begin{array}{ll}
R(x)=\frac{\sqrt{\Delta t}}{\tanh \left(\frac{x+K}{\sqrt{\Delta t}}\right)}, & K=\sqrt{\Delta t} \tanh ^{-1}(h \sqrt{\Delta t}) \\
W_{n}^{\prime}=-\frac{R(x)}{\Delta t}\left(W_{n}-C_{n-1}(x)\right), & W_{n}(0)=g\left(t_{n}\right) .
\end{array}
$$

The function $W_{n}$ is solution of well defined initial value problem and may be considered available. The free boundary $S_{n}$ is determined such that the triple $C_{n}$, 
$V_{n}, S_{n}$ simultaneously satisfies (3.23), (3.24) and (3.27). Elimination of $C_{n}$ and $V_{n}$ from (3.24) and (3.27) shows that $S_{n}$ must be a root of the scalar equation

$$
\sigma_{n}(x) \doteq \frac{x-S_{n-1}}{\Delta t}-f\left(\frac{W_{n}(x)-q R(x)\left(x-S_{n-1}\right) / \Delta t}{1+R(x)\left(x-S_{n-1}\right) / \Delta t}\right)=0 .
$$

Given $S_{n}$, we set

$$
C_{n}\left(S_{n}\right)=\frac{W_{n}\left(S_{n}\right)-R\left(S_{n}\right) \dot{S}_{n} q}{1+R\left(S_{n}\right) \dot{S}_{n}},
$$

so that

$$
\dot{S}_{n} \doteq \frac{S_{n}-S_{n-1}}{\Delta t}=f\left(C_{n}\left(S_{n}\right)\right)
$$

and

$$
C_{n}^{\prime}\left(S_{n}\right)=V_{n}\left(S_{n}\right)=-\dot{S}_{n} \frac{W_{n}\left(S_{n}\right)+q}{1+R\left(S_{n}\right) \dot{S}_{n}} .
$$

Thus, the triple $\left\{C_{n}\left(S_{n}\right), V_{n}\left(S_{n}\right), S_{n}\right\}$ is an exact solution of (3.23), (3.24) and (3.27). We remark that depending on $\Delta t$ the functional $\sigma_{n}(x)$ may have a root smaller than $S_{n-1}$. Such a root would correspond to a negative concentration $C_{n}\left(S_{n}\right)$ and is not admissible. We shall therefore agree to choose for $S_{n}$ the smallest root of $\sigma_{n}(x)=0$ on $\left(S_{n-1}, \infty\right)$. Such a root will be soon to exist.

Once $S_{n}$ has been determined, one can find $V_{n}$ by integrating backward over $\left[0, S_{n}\right)$ the equation

$$
V_{n}^{\prime}=\frac{1}{\Delta t}\left(R(x) V_{n}+W_{n}(x)-C_{n-1}(x)\right),
$$

with $V_{n}\left(S_{n}\right)$ given by (3.33). The concentration $C_{n}(x)$ at time level $t_{n}$ is obtained from (3.27). Finally, $C_{n}(x)$ is extended over $\left[S_{n}, \infty\right)$ as $C^{1}$ linear function, because $C_{n+1}(x)$ will be computed in $\left[0, S_{n+1}\right]$, with $S_{n+1}>S_{n}$, as the solution of an ODE depending on $C_{n}$. For the initial concentration we shall use

$$
C_{0}(x)=-h\left(1-c^{*}\right) x+c^{*}
$$

Lemma 3.1. There exists a solution $S_{n}$ of $(3.30)$ on $\left(S_{n-1}, \infty\right)$ and $S_{n}-S_{n-1}<$ $f(1) \Delta t$. The function $C_{n}$ satisfies $0<C_{n}<1$ on $\left[0, S_{n}\right]$ and $C_{n}^{\prime}<0$ on $\left[S_{n}, \infty\right)$. 
Proof. We note that $C_{0}\left(S_{0}\right)=c^{*} \in(0,1)$ and $C_{0}^{\prime}=-h\left(1-c^{*}\right)<0$. Let us proceed by induction and assume the result valid for $n-1$. Integrating (3.29) we have

$$
W_{n}(x)=\frac{1}{\sinh \left(\frac{x+K}{\sqrt{\Delta t}}\right)}\left[g\left(t_{n}\right) \sinh \left(\frac{K}{\sqrt{\Delta} t}\right)+\frac{1}{\sqrt{\Delta t}} \int_{0}^{x} \cosh \left(\frac{r+K}{\sqrt{\Delta t}}\right) C_{n-1}(r) d r\right],
$$

since $C_{n-1}<1$ by assumption, we get

$$
\begin{aligned}
W_{n}(x) & \leq \frac{1}{\sinh \left(\frac{x+K}{\sqrt{\Delta t}}\right)}\left[g\left(t_{n}\right) \sinh \left(\frac{K}{\sqrt{\Delta} t}\right)+\frac{1}{\sqrt{\Delta t}} \int_{0}^{x} \cosh \left(\frac{r+K}{\sqrt{\Delta t}}\right) d r\right] \\
& =\frac{1}{\sinh \left(\frac{x+K}{\sqrt{\Delta t}}\right)}\left[g\left(t_{n}\right) \sinh \left(\frac{K}{\sqrt{\Delta} t}\right)+\left(\sinh \left(\frac{x+K}{\sqrt{\Delta t}}\right)-\sinh \left(\frac{K}{\sqrt{\Delta t}}\right)\right)\right] \\
& \leq 1
\end{aligned}
$$

moreover $W_{n}\left(S_{n-1}\right)>0$. Hence the function

$$
\frac{W_{n}(x)-q R(x)\left(x-S_{n-1}\right) / \Delta t}{1+R(x)\left(x-S_{n-1}\right) / \Delta t}
$$

is less than one and positive on some interval $\left(S_{n-1}, x_{0}\right)$, vanishing on $x_{0}$. Then,

$$
\sigma_{n}\left(x_{0}\right)=\frac{x_{0}-S_{n-1}}{\Delta t}-f(0)>0,
$$

what is more

$$
\sigma_{n}\left(S_{n-1}\right)=-f\left(W_{n}\left(S_{n-1}\right)\right)<0,
$$

thus there must be a point $S_{n} \in\left(S_{n-1}, x_{0}\right)$ where $\sigma_{n}\left(S_{n}\right)=0,0<C_{n}\left(S_{n}\right)<1$ and $C_{n}^{\prime}\left(S_{n}\right)<0$. Integrating (3.27) we obtain:

$$
\begin{aligned}
0 & <C_{n}(x) \\
& =\cosh \left(\frac{x+K}{\sqrt{\Delta t}}\right)\left[\frac{C_{n}\left(S_{n}\right)}{\cosh \left(\frac{S_{n}+K}{\sqrt{\Delta t}}\right)}-\frac{1}{\sqrt{\Delta t}} \int_{S_{n}}^{x} \frac{\sinh \left(\frac{r+K}{\sqrt{\Delta t}}\right)}{\cosh ^{2}\left(\frac{r+K}{\sqrt{\Delta t}}\right)} W_{n}(r) d r\right] \\
& \leq \cosh \left(\frac{x+K}{\sqrt{\Delta t}}\right)\left[\frac{C_{n}\left(S_{n}\right)}{\cosh \left(\frac{S_{n}+K}{\sqrt{\Delta t}}\right)}-\frac{1}{\sqrt{\Delta t}} \int_{S_{n}}^{x} \frac{\sinh \left(\frac{r+K}{\sqrt{\Delta t}}\right)}{\cosh ^{2}\left(\frac{r+K}{\sqrt{\Delta t}}\right)} d r\right] \\
& =C_{n}\left(S_{n}\right) \frac{\cosh \left(\frac{x+K}{\sqrt{\Delta t}}\right)}{\cosh \left(\frac{S_{n}+K}{\sqrt{\Delta t}}\right)}+1-\frac{\cosh \left(\frac{x+K}{\sqrt{\Delta t}}\right)}{\cosh \left(\frac{S_{n}+K}{\sqrt{\Delta t}}\right)}<1 \quad \forall x \in\left[0, S_{n}\right] .
\end{aligned}
$$


Finally, from (3.31) and (3.32) we conclude that $S_{n}-S_{n-1}=f\left(C_{n}\left(S_{n}\right)\right) \Delta t<$ $f(1) \Delta t$.

Remark. $S_{n-1}$ can not be an accumulation point of the set of points that satisfy $\sigma_{n}(x)=0$ since $\sigma_{n}$ is a continuous function and $\sigma_{n}\left(S_{n-1}\right)<0$.

\section{Asymptotic behavior}

In this section we show some results about the behavior of the free boundary $s(t)$ when $t$ goes to infinity.

Let $s(t), c(x, t)$ solve problem PS. Using Green's identity we get:

$$
0=\oint_{\partial D_{t}} c(x, t) v(x, t) d x+\left(v(x, t) c_{x}(x, t)-c(x, t) v_{x}(x, t)\right) d t \quad t>0,
$$

which holds for every solution $v=v(x, t)$ of $v_{x x}+v_{t}=0$ in $D_{t}$. Thus, taking $v(x, t)=1$ we obtain

$$
0=\oint_{\partial D_{t}} c(x, t) d x+c_{x}(x, t) d t \quad t>0,
$$

which gives

$$
\begin{aligned}
0= & \int_{0}^{t} c(s(\tau), \tau) \dot{s}(\tau) d \tau-\int_{0}^{t} \dot{s}(\tau)(c(s(\tau), \tau)+q) d \tau \\
& -\int_{0}^{s(t)} c(x, t) d x-\int_{0}^{t} c_{x}(0, \tau) d \tau
\end{aligned}
$$

and then

$$
\begin{aligned}
q s(t) & =-\int_{0}^{s(t)} c(x, t) d x-\int_{0}^{t} c_{x}(0, \tau) d \tau \\
& =-\int_{0}^{s(t)} c(x, t) d x-h \int_{0}^{t} c(0, \tau) d \tau+h \int_{0}^{t} g(\tau) d \tau
\end{aligned}
$$

so

$$
s(t) \leq \frac{h}{q} \int_{0}^{t} g(\tau) d \tau \leq \frac{h}{q} G .
$$

This upper bound is independent of $f$, and since $\dot{s}(t)=f(c(s(t), t))>0$ there exists

$$
s_{\infty} \doteq \lim _{t \rightarrow \infty} s(t)
$$


The following numerical result shows these facts for the case $q=.3, h=10$, $g(t)=e^{-2 t}$ and several functions $f\left(\frac{h}{q} G=16.7\right)$. We solved the equations (3.29) and (3.34) using Runge-Kutta method implemented in Matlab as ODE45, with the step size and tolerance established by default.

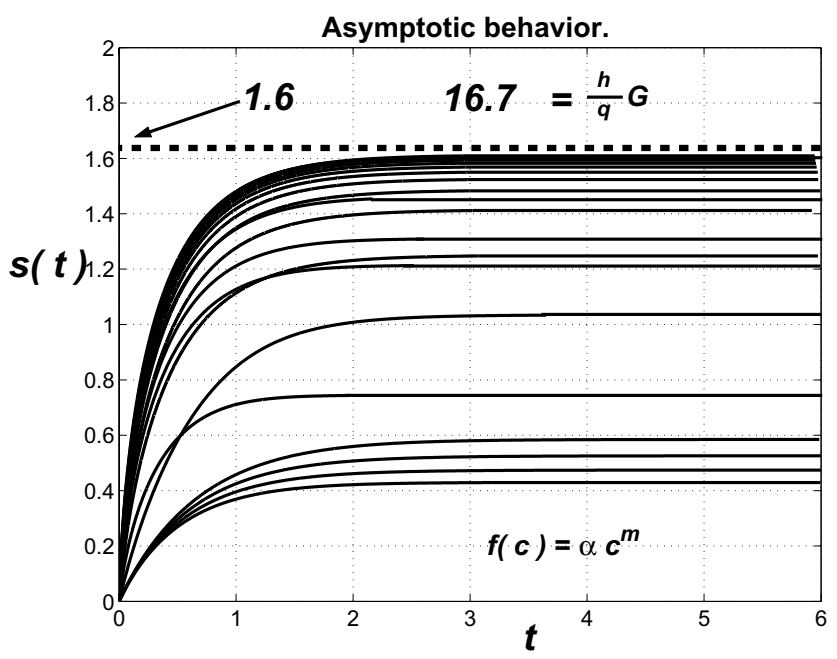

Figure 1 - Plot of the free boundaries and their asymptotic behaviors for several function $f(c)=\alpha c^{m}, \alpha>0, m>0$.

The Figure 1 shows that all the free boundaries are bounded nearly by 1.6 , so $\frac{h}{q} G$ appears to be a very large bound, and we can look for a better one. In order to do it, we will obtain two additional equations for $s$ and $c$ ( (4.40) and (4.41) below). First, taking $v(x, t)=x$ in (4.35) we have

$$
0=\oint_{\partial D_{t}} c(x, t) x d x+\left(x c_{x}(x, t)-c(x, t)\right) d t
$$

it gives

$$
\begin{aligned}
0= & \int_{0}^{t} c(s(\tau), \tau) s(\tau) \dot{s}(\tau) d \tau \\
& +\int_{0}^{t}[-(q+c(s(\tau), \tau)) \dot{s}(\tau) s(\tau)-c(s(\tau), \tau)] d \tau \\
& -\int_{0}^{s(t)} c(x, t) x d x+\int_{0}^{t} c(0, \tau) d \tau
\end{aligned}
$$




$$
\frac{q}{2} s^{2}(t)+\int_{0}^{t} c(s(\tau), \tau) d \tau=-\int_{0}^{s(t)} c(x, t) x d x+\int_{0}^{t} c(0, \tau) d \tau .
$$

Similarly, taking $v(x, t)=t-\frac{x^{2}}{2}$ we get:

$$
0=\oint_{\partial D_{t}} c(x, t)\left(t-\frac{x^{2}}{2}\right) d x+\left[\left(t-\frac{x^{2}}{2}\right) c_{x}(x, t)+x c(x, t)\right] d t
$$

thus

$$
\begin{aligned}
0= & \int_{t}^{0} \tau c_{x}(0, \tau) d \tau+\int_{0}^{t}\left[c(s(\tau), \tau)\left(\tau-\frac{s^{2}(\tau)}{2}\right) \dot{s}(\tau)\right. \\
& \left.+\left(\tau-\frac{s^{2}(\tau)}{2}\right) c_{x}(s(\tau), \tau)+c(s(\tau), \tau) s(\tau)\right] d \tau \\
& +\int_{s(t)}^{0} c(x, t)\left(t-\frac{x^{2}}{2}\right) d x
\end{aligned}
$$

and so

$$
\begin{aligned}
0= & -\int_{0}^{t} \tau c_{x}(0, \tau) d \tau-\frac{q}{6} s^{3}(t)-q \int_{0}^{t} \tau \dot{s}(\tau) d \tau \\
& +\int_{0}^{t} c(s(\tau), \tau) s(\tau) d \tau+\frac{1}{2} \int_{0}^{s(t)} x^{2} c(x, t) d x \\
& -t \int_{0}^{s(t)} c(x, t) d x .
\end{aligned}
$$

Lemma 4.1. The following equation holds:

$$
\lim _{t \rightarrow \infty} \int_{0}^{s(t)} c(x, t) d x=0 .
$$

Proof. From (4.41) we have

$$
\begin{aligned}
\int_{0}^{s(t)} c(x, t) d x= & -\frac{1}{t} \int_{0}^{t} \tau c_{x}(0, \tau) d \tau-\frac{q}{6 t} s^{3}(t)-\frac{q}{t} \int_{0}^{t} \tau \dot{s}(\tau) d \tau \\
& +\frac{1}{t} \int_{0}^{t} c(s(\tau), \tau) s(\tau) d \tau+\frac{1}{2 t} \int_{0}^{s(t)} x^{2} c(x, t) d x \\
\leq & -\frac{1}{t} \int_{0}^{t} \tau c_{x}(0, \tau) d \tau+\frac{1}{t} \int_{0}^{t} c(s(\tau), \tau) s(\tau) d \tau+\frac{s_{\infty}^{3}}{2 t} .
\end{aligned}
$$


To prove the lemma it is enough that all these terms go to zero as $t \rightarrow \infty$. In order to do it, note that from (4.37),

$$
\int_{0}^{t} c(0, \tau) d \tau \leq \int_{0}^{t} g(\tau) d \tau \leq G
$$

then, using (4.40)

$$
\int_{0}^{t} c(s(\tau), \tau) s(\tau) d \tau \leq s_{\infty} \int_{0}^{t} c(s(\tau), \tau) d \tau \leq s_{\infty} G
$$

thus

$$
\lim _{t \rightarrow \infty} \frac{1}{t} \int_{0}^{t} c(s(\tau), \tau) s(\tau) d \tau=0 .
$$

On the other hand, from (4.36)

$$
-\int_{0}^{t} c_{x}(0, \tau) d \tau=\int_{0}^{s(t)}[q+c(x, t)] d x>0
$$

and from (1.2)

$$
-\int_{0}^{\infty} c_{x}(0, \tau) d \tau=h\left(G-\int_{0}^{\infty} c(0, \tau) d \tau\right)<\infty .
$$

And integration by parts gives

$$
\begin{aligned}
& \lim _{t \rightarrow \infty} \frac{1}{t} \int_{0}^{t} \tau c_{x}(0, \tau) d \tau \\
& \quad= \lim _{t \rightarrow \infty}\left[\int_{0}^{t} c_{x}(0, \tau) d \tau-\frac{1}{t} \int_{0}^{t}\left(\int_{0}^{\tau} c_{x}\left(0, \tau^{\prime}\right) d \tau^{\prime}\right) d \tau\right] \\
& \quad=0
\end{aligned}
$$

Where in the last equality we have used (4.43), (4.44) and the L'Hôpital's rule.

Lemma 4.2. Assume that $f(c)=\alpha c, \alpha>0$. Then the explicit formula holds:

$$
s_{\infty}=\sqrt{\left(\frac{1}{h}+\frac{1}{\alpha q}\right)^{2}+\frac{2}{q} G}-\left(\frac{1}{h}+\frac{1}{\alpha q}\right) .
$$


Proof. Cancelling $\int_{0}^{t} c(0, \tau) d \tau$ from (4.37) and (4.40) we obtain

$$
\begin{aligned}
\frac{q}{2} s^{2}(t)+\frac{q}{h} s(t)= & -\int_{0}^{s(t)}\left(\frac{1}{h}+x\right) c(x, t) d x \\
& +\int_{0}^{t} g(\tau) d \tau-\int_{0}^{t} c(s(\tau), \tau) d \tau,
\end{aligned}
$$

and since $c(s(\tau), \tau)=\frac{1}{\alpha} \dot{s}(\tau)$, we get

$$
\frac{q}{2} s^{2}(t)+\left(\frac{1}{\alpha}+\frac{q}{h}\right) s(t)=\int_{0}^{t} g(\tau) d \tau-\int_{0}^{s(t)}\left(\frac{1}{h}+x\right) c(x, t) d x,
$$

observe that

$$
\int_{0}^{s(t)}\left(\frac{1}{h}+x\right) c(x, t) d x \leq\left(\frac{1}{h}+s_{\infty}\right) \int_{0}^{s(t)} c(x, t) d x,
$$

and then by (4.42) we get

$$
\frac{q}{2} s_{\infty}^{2}+\left(\frac{1}{\alpha}+\frac{q}{h}\right) s_{\infty}=G
$$

Theorem 4.1. The following statement is true

$$
\sup _{f} s_{\infty}=\sqrt{\frac{1}{h^{2}}+\frac{2}{q} G}-\frac{1}{h} .
$$

The supremum is taken on the set of the functions $f$ belonging to $C^{1}(0,1]$ satisfying $f^{\prime}(c)>0$ in $(0,1]$ and $f(0)=0$.

Proof. Proceeding as in (4.46), we have

$$
\begin{aligned}
s^{2}(t)+\frac{2}{h} s(t)-\frac{2}{q} \int_{0}^{t} g(\tau) d \tau= & -\frac{2}{q} \int_{0}^{s(t)}\left(\frac{1}{h}+x\right) c(x, t) d x \\
& -\int_{0}^{t} c(s(\tau), \tau) d \tau \leq 0,
\end{aligned}
$$

as $t \rightarrow \infty$ we get

$$
s_{\infty}^{2}+\frac{2}{h} s_{\infty}-\frac{2}{q} G \leq 0
$$

thus

$$
s_{\infty} \leq \sqrt{\frac{1}{h^{2}}+\frac{2}{q} G}-\frac{1}{h},
$$

and (4.47) follows taking $\alpha \rightarrow \infty$ in (4.45). 


\section{Conclusions and final comment}

In this paper the main result is the asymptotic behavior of the free boundary. We remark that the upper bound (4.47) should be very useful for real applications, where the function $f$ is a priori unknown and a estimate of $s_{\infty}$ is needed. From the physical point of view we emphasize that the bound of the free boundary does not depend on the function $f$. That means that this behavior of the free boundary holds for all kind of homogeneous polymers with constant diffusivity. For the case of two dimensional space variable we expect to have bounds for the free boundary that do not depend on $f$. This will be the subject of future work.

Acknowledgement. This paper has been sponsored by the grant Secyt-UNC and CONICET and Marcos Gaudiano was sponsored by an scholarship of CONICET.

\section{REFERENCES}

[1] G. Astarita and G.C. Sarti, A Class of Mathematical Models for Sorption of Swelling Solvents in Glassy Polymers. Polymer Engineering and Science, 18(5) (1978), 388-395.

[2] A. Fasano, G.H. Meyer and M. Primicerio, On a Problem in the Polymer Industry: Theoretical and Numerical Investigation of Swelling. S.I.A.M., 17(4) (1986), 945-960.

[3] A. Comparini and R. Ricci, On the Swelling of a Glassy Polymer in Contact with a Well-stirred Solvent. Mathematical Methods in the Applied Sciences, 7 (1985), 238-250.

[4] A. Comparini, R. Ricci and C. Turner, Penetration of a solvent into a non-homogeneous polymer. Meccanica, 23 (1988), 75-80.

[5] D. Andreucci and R. Ricci, A free boundary problem arising from sorption of solvent in glassy polymers. Quarterly of applied mathematics, 44 (1987), 649-657.

[6] G.H. Meyer, One-Dimensional Parabolic Free Boundary Problems. S.I.A.M. Review, 19(1) (1977), 17-33.

[7] M. Gaudiano, C. Turner and T. Godoy, Technical Report: Free boundary problems in Industry: Diffusion of a solvent into a polymer. (http://www.famaf.unc.edu.ar/series/AMat2005.htm). 